\title{
Assessment of the MANTA closure device in transfemoral transcatheter aortic valve replacement: a single-centre observational study
}

\author{
J. Halim • L. Missault • M. Lycke · J. Van der Heyden
}

Published online: 27 July 2020

(c) The Author(s) 2020

\begin{abstract}
Objectives The present study aims to evaluate the efficacy and safety of the MANTA vascular closure device (VCD) (Teleflex, Morrisville, NC, USA) in transfemoral transcatheter aortic valve replacement (TF-TAVR).

Background To close the femoral artery in TF-TAVR a VCD is the treatment of choice. Data involving suture-based VCDs have been extensive. Although scarce, results on the MANTA device are promising. There is no consensus yet as to whether the MANTA device is associated with fewer access-site-related vascular/bleeding complications when compared to suture-based VCDs.

Methods In this prospective single-arm study, performed at a single centre, a total of 73 patients eligible for TF-TAVR were included and consecutively treated with the MANTA device.

Results Access-site-related vascular complications were seen in $13.7 \%$ of patients treated with the MANTA device. In this group of patients only minor vascular complications were observed. Access-site-related bleeding complications were rare $(6.8 \%)$, and device failure was seen in $13.7 \%$ of the patients.

Conclusions This single-centre study confirms that use of the MANTA device in TF-TAVR is feasible with an acceptable rate of access-site-related complications and no major vascular complications.
\end{abstract}

Keywords Vascular access · Complication . Transcatheter aortic valve replacement · Vascular closure device

J. Halim $(\bowtie) \cdot$ L. Missault · M. Lycke $\cdot$ J. Van der Heyden Department of Cardiology, Sint-Jan Hospital, Bruges, Belgium

jonathan.halim@azsintjan.be

\section{Introduction}

Transcatheter aortic valve replacement (TAVR) has emerged as the gold standard treatment for symptomatic patients at high surgical risk. While the complication rate has decreased as a result of the operators' growing expertise and knowledge, as well as new valve technology, TAVR is still associated with a significant complication rate [1].

More specifically, vascular complications at the access site for transfemoral (TF)-TAVR still remain a problem. These complications are observed in $5-20 \%$ of patients and have a significant impact on clinical outcomes [2]. During the early years of TFTAVR, the femoral artery was closed surgically. More recently, this method has been replaced by use of a vascular closure device (VCD).

\section{What's new}

- Access-site-related vascular and bleeding complications in transfemoral transcatheter aortic valve replacement (TF-TAVR) remain a problem with a significant impact on clinical outcomes.

- The MANTA vascular closure device (VCD) is efficacious and safe for use in TF-TAVR. Importantly, major vascular complications did not occur and major bleeding complications were extremely rare when using this device.

- A randomised controlled trial comparing the MANTA device with suture-based VCDs is needed to determine which device is associated with the lowest risk of access-site-related complications. 
The most commonly used VCDs for TF-TAVR are suture-based, e.g. the ProGlide and the Prostar XL (Abbott Vascular Inc., Santa Clara, CA, USA). The efficacy of these VCDs has been extensively reported in previous studies [3]. The MANTA VCD (Teleflex, Morrisville, NC, USA) is a collagen-based device that has shown promising results in TF-TAVR [4-9]. However, data regarding the vascular complication rate in comparison to the well-known suture-based VCDs are scarce.

The primary aim of this study is to assess accesssite-related complications in TF-TAVR patients treated by the MANTA device. Our hypothesis is that the MANTA device is associated with a low risk of major vascular and major bleeding complications according to the Valve Academic Research Consortium (VARC)2 definitions [10].

\section{Methods}

\section{Study design}

A total of 73 patients with severe aortic stenosis scheduled for TF-TAVR were consecutively included between July 2018 and August 2019 at the Sint-Jan Hospital in Bruges, Belgium. Informed consent was obtained in accordance with the Declaration of Helsinki. Multi-detector computed tomography was performed according to the TAVR protocol, using dedicated software (3mensio, Pie Medical Imaging, Maastricht, The Netherlands). Initially, suture-based VCDs were used at our centre. However, since July 2018 the MANTA device has been utilised. Starting then and for the purposes of this trial, data from patients treated with the MANTA device were collected prospectively. Two TAVR operators with prior experience in using the MANTA device performed the vascular closure.

\section{Procedure details}

In patients not taking antiplatelet medication, acetylsalicylic acid was started prior to the procedure. Additional clopidogrel was started on the morning of the procedure. Novel oral anticoagulant therapy was discontinued $24 \mathrm{~h}$ before the procedure. Oral anticoagulant therapy was continued, aiming for an International Normalised Ratio level of 2-2.5.

All procedures were performed with the patient under conscious sedation. Puncture of the femoral artery was performed with either fluoroscopic or ultrasound guidance. During the procedure, heparin was given and an activated clotting time between 250 and $300 \mathrm{~s}$ was targeted. The implanted bioprosthetic valves were either the CoreValve Evolut R/PRO (Medtronic, Minneapolis, MN, USA), the ACURATE neo (Boston Scientific, Natick, MA, USA) or the LOTUS Edge (Boston Scientific). In patients treated with a CoreValve a 16-French (Fr) InLine sheath or a 20-
Fr Sentrant introducer sheath was used. In patients treated with an ACURATE neo a 14-Fr iSLEEVE or a small LOTUS introducer sheath was used. A large LOTUS introducer sheath was used for implantation of the LOTUS Edge. After valve implantation, in cases where the activated clotting time was above $200 \mathrm{~s}$, protamine was given before vascular closure.

\section{MANTA device}

The MANTA closure device is a collagen-based technology available in two sizes: $14 \mathrm{Fr}$ and $18 \mathrm{Fr}$. It can be used for sheath sizes ranging from 10 to $14 \mathrm{Fr}$ and from 15 to $22 \mathrm{Fr}$ respectively. A detailed description of the MANTA device has been published previously [11]. In summary, it consists of multiple components: an 8-Fr puncture site dilator, a dedicated sheath, a closure unit and a delivery system. The site dilator and device sheath have centimetre markers. After successfully puncturing the femoral artery, a standard 6-Fr sheath is removed and the site dilator is pushed over the guidewire to measure the distance from skin level to the endoluminal space of the vessel. When vascular closure is initiated, the access sheath is removed and replaced by the MANTA sheath. The closure unit is connected with the MANTA sheath and both are withdrawn up to the predetermined deployment level. The toggle is then released, and the device is withdrawn with the colour code of the tension gauge serving as an indicator of whether sufficient pulling force is being applied. A blue tamper tube is then advanced along the suture line to position the radiopaque lock over the suture knot to secure the collagen-anchor sandwich. When haemostasis has been achieved the guidewire is removed and the suture is cut at skin level. Importantly, unlike the suture-based VCDs a pre-closure technique is not necessary [12]. Further, it may take 6 months until the components of the MANTA device are resorbed.

\section{Femoral artery evaluation: accessibility and closure device success}

Multi-detector computed tomography was performed to assess the iliofemoral arteries for vascular access. The vessel size, tortuosity, the amount of calcification and the minimum lumen diameter were analysed. Notably, a sheath to femoral artery ratio of $\geq 1.05$ is associated with a higher risk of vascular complications and 30-day mortality [13, 14].

Haemostasis success after use of the MANTA device was evaluated by use of rotational angiography after vascular closure. This meant a dynamic evaluation of the closure site from the 30-degree right anterior oblique view to the 30 -degree left anterior oblique view with administration of $20 \mathrm{cc}$ contrast medium. If leakage was present it could be classified as mild (minor extravasation of contrast without external bleeding), moderate (continuous extravasation with moder- 
ate external bleeding) or severe (continuous extravasation with significant external bleeding). If leakage occurred balloon dilatation was performed. Hereafter, rotational angiography was repeated and if leakage persisted a covered Gore Viabahn stent (Gore Medical, Flagstaff, AZ, USA) was placed.

Table 1 Baseline characteristics

\begin{tabular}{|c|c|}
\hline & $(n=73)$ \\
\hline Age (years) & $84.0 \pm 5.48$ \\
\hline Men & $40(54.8)$ \\
\hline BMI & $26.4 \pm 4.25$ \\
\hline NYHA 3 or 4 & $24(32.9)$ \\
\hline Euroscore II & $4.6 \pm 3.79$ \\
\hline$L V E F<40 \%$ & $5(6.8)$ \\
\hline Aortic valve MG & $43.1 \pm 15.76$ \\
\hline AVA & $0.73 \pm 0.20$ \\
\hline Antiplatelet therapy & $42(57.5)$ \\
\hline Anticoagulant therapy & $23(31.5)$ \\
\hline \multicolumn{2}{|l|}{ Femoral artery characteristics } \\
\hline Minimum arterial diameter (MANTA side) (mm) & $7.2 \pm 1.49$ \\
\hline \multicolumn{2}{|l|}{ Calcification (MANTA side): } \\
\hline - None & $26(35.6)$ \\
\hline - Mild & $32(43.9)$ \\
\hline - Moderate & $9(12.3)$ \\
\hline -Severe & $6(8.2)$ \\
\hline \multicolumn{2}{|l|}{ Procedural characteristics } \\
\hline \multicolumn{2}{|l|}{ Device system: } \\
\hline \multicolumn{2}{|l|}{ - Evolut PRO } \\
\hline$-23 \mathrm{~mm}$ & $1(1.4)$ \\
\hline$-26 \mathrm{~mm}$ & $7(9.6)$ \\
\hline$-29 \mathrm{~mm}$ & $16(21.9)$ \\
\hline \multicolumn{2}{|l|}{ - Evolut $R$} \\
\hline$-34 \mathrm{~mm}$ & $23(31.5)$ \\
\hline \multicolumn{2}{|l|}{ - ACURATE neo } \\
\hline - small & $2(2.7)$ \\
\hline - medium & $6(8.2)$ \\
\hline- large & $17(23.3)$ \\
\hline \multicolumn{2}{|l|}{ - Lotus Edge } \\
\hline$-27 \mathrm{~mm}$ & $1(1.4)$ \\
\hline \multicolumn{2}{|l|}{ MANTA size: } \\
\hline$-14 \mathrm{Fr}$ & $0(0)$ \\
\hline$-18 \mathrm{Fr}$ & $73(100)$ \\
\hline \multicolumn{2}{|l|}{ Femoral sheaths: } \\
\hline$-14 \mathrm{Fr}$, iSLEEVE & $13(17.8)$ \\
\hline$-16 \mathrm{Fr}$, InLine sheath & $45(61.6)$ \\
\hline -20Fr, Sentrant sheath & $3(4.1)$ \\
\hline -Small LOTUS & $11(15.1)$ \\
\hline -Large LOTUS & $1(1.4)$ \\
\hline Sheath to femoral artery ratio & $1.03 \pm 0.27$ \\
\hline \multicolumn{2}{|c|}{$\begin{array}{l}\text { Data presented as number }(\%) \text { or mean } \pm \text { SD } \\
S D \text { standard deviation, } B M I \text { body mass index, NYHA New York Heart } \\
\text { Association, } L V E F \text { left ventricular ejection fraction, } M G \text { mean gradient, } \\
A V A \text { aortic valve area, } F r \text { French }\end{array}$} \\
\hline
\end{tabular}

\section{Study endpoints}

The primary endpoint of this study was the evaluation of device failure and vascular/bleeding complications at the access site according to the VARC-2 definitions. Secondary endpoints included mortality and vascular/bleeding complications at the access site at 30day follow-up.

\section{Statistical analysis}

Statistical analysis included descriptive statistics. Categorical variables were presented as frequencies and percentages and continuous variables as mean \pm standard deviation (SD). All analyses were conducted with SPSS v.26 (IBM, Chicago, IL, USA).

\section{Results}

\section{Baseline characteristics}

A total of 73 patients were included in our study and treated with the MANTA device. Baseline characteristics are shown in Tab. 1. The mean age of this group of patients was $84 \pm 5.48$ years and the majority of these patients were men $(54.8 \%)$. Antiplatelet therapy was used in more than half of the patients. Evolut R/PRO was implanted in more than half of the cases $(64.4 \%)$.

Mean minimal femoral arterial diameter at the access site was $7.2 \pm 1.49 \mathrm{~mm}$ and mean sheath to femoral artery ratio was $1.03 \pm 0.27$. In all patients the MANTA 18-Fr device was used for femoral artery closure.

\section{Outcomes}

\section{Primary endpoint}

Outcomes of device failure and vascular/bleeding complications at the access site are shown in Tab. 2. Ten patients (13.7\%) had device failure. In these 10 patients use of a covered Gore Viabahn stent was necessary. Details of the femoral artery and introducer sheath used in this group of patients are summarised

Table 2 Primary and secondary endpoints

\begin{tabular}{|l|c|}
\hline Device failure & $(n=73)$ \\
\hline All vascular complications (major + minor) & $10(13.7)$ \\
\hline Major vascular complications & $10(13.7)$ \\
\hline Minor vascular complications & $0(0)$ \\
\hline Surgical closure & $10(13.7)$ \\
\hline All bleeding complications (major + minor) & $0(0)$ \\
\hline Major bleeding complications & $5(6.8)$ \\
\hline Minor bleeding complications & $1(1.4)$ \\
\hline 30-day mortality & $4(5.5)$ \\
\hline 30-day vascular/bleeding complications & $4(5.5)$ \\
\hline Data presented as number (\%) & $1(1.4)$ \\
\hline
\end{tabular}


Table 3 Vessel and sheath characteristics of patients with device failure

\begin{tabular}{l|l|}
\hline $\begin{array}{l}\text { Femoral artery characteristics } \\
\text { Minimum arterial diameter (MANTA side) }(\mathrm{mm})\end{array}$ & $(n=10)$ \\
\hline $\begin{array}{l}\text { Calcification (MANTA side) } \\
\text { - None }\end{array}$ & $7.04 \pm 0.71$ \\
\hline - Mild & $5(50)$ \\
\hline - Moderate & $4(40)$ \\
\hline Procedural characteristics & $1(10)$ \\
\hline $\begin{array}{l}\text { Femoral sheaths: } \\
\text { - 14Fr, iSLEEVE }\end{array}$ \\
\hline - 16Fr, InLine sheath & \\
\hline - Small Lotus & $5(50)$ \\
\hline Sheath to femoral artery ratio & $3(30)$ \\
\hline $\begin{array}{l}\text { Data presented as number (\%) or mean } \pm \text { SD } \\
\text { FrFrench }\end{array}$ & $2(20)$ \\
\hline
\end{tabular}

in Tab. 3. No major differences could be seen when compared to the femoral artery and introducer sheath characteristics of the entire study population.

Vascular complications were present in 10 patients (13.7\%). However, major vascular complications did not occur. Minor vascular complications were present in 10 patients $(13.7 \%)$, in whom balloon dilatation did not result in haemostasis; subsequently, covered stent implantation was performed. Additional surgery was not needed.

Bleeding complications occurred in five patients (6.8\%), but none were life-threatening or disabling. One patient experienced a major bleeding complication at the groin, resulting in a drop in haemoglobin of greater than $3.0 \mathrm{~g} / \mathrm{dl}$ and the need for a blood cell transfusion. A minor bleeding complication was present in four patients, in whom prolonged manual compression led to an access site haematoma and a slight fall in haemoglobin level.

\section{Secondary endpoint}

Late major bleeding complications were seen in one patient at 30-day follow-up. In this patient a haematoma at the femoral access site as well as haematuria occurred. Three units of packed red blood cells were transfused. Late vascular complications did not occur.

Thirty-day mortality was $5.5 \%(n=4)$. One patient developed cardiogenic shock during hospitalisation, which led to cardiac arrest. Echocardiography in the acute setting did not reveal structural abnormalities. The second patient underwent a valve-in-valve intervention (previous Solo stentless bioprosthetic aortic valve implantation; LivaNova, London, UK), whereby implantation of the new bioprosthetic valve led to occlusion of the left main coronary artery, resulting in cardiac arrest and death. The third patient experienced a periprocedural myocardial infarction. Shortly hereafter, cardiogenic shock resulted in death. Lastly, one patient died following complications resulting from a periprocedural cerebrovascular accident.

\section{Discussion}

In this prospective single-centre study evaluating the MANTA device in TF-TAVR, no major vascular complications were encountered. A major bleeding complication occurred in one patient (1.4\%). Minor vascular and minor bleeding complications were seen in $13.7 \%$ and $5.5 \%$ of the patients respectively. Device failure occurred in $13.7 \%$ of the patients. At 30-day follow-up, mortality and the rate of vascular/bleeding complications were low.

Van Mieghem et al. [4] performed a single-arm prospective study including 50 patients, in whom the use of the MANTA device in TF-TAVR and high-risk percutaneous coronary intervention was associated with rapid haemostasis success and a low vascular complication rate. Major vascular and major bleeding complications were each seen in $2 \%$ of the patients. Minor vascular complications were not observed. De Palma et al. [5] evaluated the MANTA device in TFTAVR in a prospective observational study and revealed similarly promising results with major vascular and major bleeding complications each being present in only $1.1 \%$ of the patients. Minor vascular and minor bleeding complications occurred in $4.5 \%$ and $11.2 \%$ of the patients respectively. Wood et al. [6] assessed the MANTA device prospectively in 341 patients undergoing TAVR or endovascular aneurysm repair and noted rapid haemostasis success and a low rate of major vascular and major bleeding complications $(2.3 \%$ and $1.1 \%$ respectively). VARC-2-defined minor vascular and minor bleeding complications were not reported. In the study of Biancari et al. [7], higher rates of major vascular and major bleeding complications $(9.3 \%$ and $15.9 \%$ ) were observed. Minor vascular complications were present in $3.7 \%$ of their patients. Minor bleeding complications were not noted. Moriyama et al. [8] reported vascular complications in $8 \%$ of their patients. Major and minor bleeding complications were seen in $10 \%$ and $4 \%$ of the patients. Moccetti et al. [9] also reported acceptable rates of major vascular and major bleeding complications ( $9 \%$ and $3 \%$ respectively) when using the MANTA device: $5 \%$ of the patients had a minor vascular complication and $7 \%$ of the patients a minor bleeding complication. Importantly, MANTAassociated vascular complications were seen more often in patients with significant peripheral disease and narrower $(<6 \mathrm{~mm})$ femoral arteries.

To date, there is no consensus as to whether the MANTA device is associated with fewer VARC-2 access-site-related major vascular/bleeding complications when compared to the suture-based VCDs. Biancari et al. [7] retrospectively compared the MANTA device with the ProGlide device in 222 patients. There were no significant differences in VARC-2 vascular and bleeding complications between these two de- 
vices. However, in the study by De Palma et al., fewer major bleeding complications were seen with the MANTA device than with the Prostar XL device [5]. In the above-mentioned trial by Moriyama et al., including 325 patients, these two VCDs were compared retrospectively by use of a propensity-matched analysis. In the MANTA group, there were significantly fewer bleeding complications-importantly, mainly as a result of the lower number of major bleeding complications. Access-site-related and access-related vascular injuries were also observed less frequently in the MANTA group. The incidence of major vascular complications was, however, comparable between these two groups [8].

The present study revealed a similar trend towards a low rate of access-site-related major vascular and major bleeding complications when using the MANTA device. No major vascular complications were observed.

The presence of minor vascular complications was slightly higher than has been reported previously. In the study of Van Mieghem et al. [4], for instance, where iliofemoral angiography was performed after vascular closure, minor vascular complications were not encountered and only three patients had signs of contrast extravasation. However, it should be noted that in the studies of De Palma et al. and Biancari et al. iliofemoral angiography was not performed systematically after vascular closure, and this was not mentioned in the study of Moriyama et al. This could be a possible explanation for the differences in minor vascular complications reported in these studies.

In addition, in the present study haemostasis success was consistently determined by rotational angiography in contrast with other studies where this was done by external inspection. A covered stent was placed due to persistent leakage without the presence of a major bleeding or signs of visceral ischaemia. Hence, a lower threshold for the use of balloon dilatation and covered stent placement could have been set compared to that in other studies.

The prevalence of vascular and bleeding complications in this study was largely comparable with that reported in previous studies and confirms that the MANTA device is a feasible option in TF-TAVR patients [2, 15]. Notably, a low rate of major accesssite-related complications was observed. The MANTA device shows promising results and as it demands a shorter learning curve in comparison with the suture-based VCDs there is a possibility that the MANTA device will become the preferred option for vascular closure in TF-TAVR. However, future randomised controlled studies comparing the MANTA device with the suture-based VCDs represent a necessary first step to elucidate which type of VCD is associated with the lowest risk of access-site-related complications in TFTAVR patients.

\section{Limitations}

This study has several limitations. First, the sample size is relatively small and the study has a non-randomised design. Second, the performance of the 14-Fr MANTA device cannot be assessed because the 18-Fr device was used in all patients undergoing TF-TAVR. However, there was no selection bias, with the enrolment of consecutive patients treated with the MANTA device.

\section{Conclusion}

The use of the MANTA device, which is a collagenbased VCD, is feasible in TF-TAVR patients and is associated with acceptable rates of vascular and bleeding complications at the access site. One major bleeding complication and no major vascular complications were seen after use of the MANTA device.

Conflict of interest J. Halim, L. Missault, M. Lycke and J. Van der Heyden declare that they have no competing interests.

Open Access This article is licensed under a Creative Commons Attribution 4.0 International License, which permits use, sharing, adaptation, distribution and reproduction in any medium or format, as long as you give appropriate credit to the original author(s) and the source, provide a link to the Creative Commons licence, and indicate if changes were made. The images or other third party material in this article are included in the article's Creative Commons licence, unless indicated otherwise in a credit line to the material. If material is not included in the article's Creative Commons licence and your intended use is not permitted by statutory regulation or exceeds the permitted use, you will need to obtain permission directly from the copyright holder. To view a copy of this licence, visit http://creativecommons.org/licenses/by/4.0/.

\section{References}

1. Fearon WF, Kodali S, Doshi D, et al. Outcomes after transfemoral transcatheter aortic valve replacement: a comparison of the randomized PARTNER (Placement of AoRTic TraNscathetER Valves) trial with the NRCA (Nonrandomized Continued Access) registry. JACC Cardiovasc Interv. 2014;11:1245-51.

2. GenereuxP,WebbJG, SvennsonLG, etal. Vascularcomplications after transcatheter aortic valve replacement insights from the PARTNER (Placement of AoRTic TraNscathetER Valve) trial. JAm Coll Cardiol. 2012;60:1043-52.

3. Maniotis C, Andreou C, Karalis I, Koutouzi G, Agelaki M, Koutouzis M. A systematic review on the safety of Prostar XL versus ProGlide after TAVR and EVAR. Cardiovasc Revasc Med. 2017;2:145-50.

4. Van Mieghem NM, Latib A, Van der Heyden J, et al. Percutaneous plug-based arteriotomy closure device for large-bore access: a multicenter prospective study. JACC Cardiovasc Interv. 2017;10:613-9.

5. De Palma R, Settergen M, RuckA, Linder R, Saleh N. Impact of percutaneous femoral arteriotomy closure using the MANTA $^{\mathrm{TM}}$ device on vascular and bleeding complications after transcatheter aortic valve replacement. Catheter Cardiovasc Interv. 2018;5:954-61. 
6. Wood DA, Krajcer Z, Sathananthan J, et al. Pivotal clinical study to evaluate the safety and effectiveness of the MANTA percutaneous vascular closure device. Catheter Cardiovasc Interv. 2019;7:e7258.

7. Biancari F, Romppanen H, Savontaus M, et al. MANTA versus ProGlide vascular closure devices in transfemoral transcatheter aortic valve implantation. Int J Cardiol. 2018;263:29-31.

8. Moriyama N, Lindstrom L, Laine M. Propensity-matched comparison of vascular closure devices after transcatheter aortic valve replacement using MANTA versus ProGlide. EuroIntervention. 2019;15:1558-65.

9. Moccetti F, Brinkert M, Seelos R, et al. Insights from a multidisciplinary introduction of the MANTA vascular closure device. JACC Cardiovasc Interv. 2019;17:1720-36.

10. Kappetein AP, Head SJ, Généreux P, et al. Updated standardized endpoint definitions for transcatheter aortic valve implantation: the Valve Academic Research Consortium-2 consensus document. JAm Coll Cardiol. 2012;60:1438-54.
11. Van Gils L, De Jaegere PP, Roubin G, Van Mieghem NM. The MANTA vascular closure device: a novel device for large-bore vessel closure. JACC Cardiovasc Interv. 2016;11:1195-6.

12. Toggweiler S, Leipsic J, Binder RK, et al. Management of vascular access in transcatheter aortic valve replacement: part 1: basic anatomy, imaging, sheaths, wires, and access routes. JACC Cardiovasc Interv. 2013;6:643-53.

13. Hayashida K, Lefevre T, Chevalier B, et al. Transfemoral aortic valve implantation new criteria to predict vascular complications. JACC Cardiovasc Interv. 2011;8:851-8.

14. Toggweiler S, Gurvitch R, Leipsic J, et al. Percutaneous aortic valve replacement: vascular outcomes with a fully percutaneous procedure. J Am Coll Cardiol. 2012;59:113-8.

15. Généreux P, Head S, Van Mieghem NM, et al. Clinical outcomes after transcatheter aortic valve replacement using Valve Academic Research Consortium definitions: a weighted meta-analysis of 3,519 patients from 16 studies. JAm Coll Cardiol. 2012;12:1043-52. 